\title{
Erratum: Extremely Low Electron-ion Temperature Relaxation Rates in Warm Dense Hydrogen: Interplay between Quantum Electrons and Coupled Ions [Phys. Rev. Lett. 122, 015001 (2019)]
}

Qian Ma, Jiayu Dai®, Dongdong Kang, M. S. Murillo, Yong Hou, Zengxiu Zhao, and Jianmin Yuan

(Q) (Received 12 August 2019; published 28 August 2019)

DOI: 10.1103/PhysRevLett.123.099901

$b_{c}$ in the coupled Gericke, Murillo, and Schlanges (GMS) model in this Letter has a typographical error. The correct $b_{c}$ is $Z e^{2} / \frac{1}{2}\left(k_{B} T_{e}+k_{B} T_{i}\right)$ and not $\frac{1}{2} Z e^{2} /\left(k_{B} T_{e}+k_{B} T_{i}\right)$. To introduce the coupling between electrons and ions, we define $b_{c}=Z e^{2} / \frac{1}{2}\left(k_{B} T_{e}+k_{B} T_{i}\right)$ instead of $b_{c}=b_{0}=Z e^{2} / k_{B} T_{e}$, typically used in the GMS model $L_{\mathrm{GMS}}=\frac{1}{2} \ln \left\{1+\left[\left(\lambda_{D}^{2}+R_{i}^{2}\right) /\left(\Lambda^{2} / 8 \pi+b_{c}^{2}\right)\right]\right\}$. 